\title{
Informações nutricionais e de saúde disponibilizadas aos consumidores por restaurantes comerciais, tipo fast food e full service
}

\author{
Nutritional and health information released to consumers by commercial fast food and full service restaurants
}

Vanessa MAESTRO ${ }^{1}$, Elisabete SALAY ${ }^{1 *}$

\begin{abstract}
Resumo
O objetivo principal deste estudo foi identificar e caracterizar o oferecimento de informação nutricional e de saúde em restaurantes comerciais do município de Campinas-SP. Com a utilização de questionários previamente testados, foram entrevistados, entre outubro e dezembro de 2005, 20 gerentes de restaurantes do tipo fast food e 94 do tipo full service, localizados nas cinco regiões de Campinas. Após a coleta das informações, foi construído um banco de dados utilizando o software Excel. Para o tratamento estatístico, utilizou-se o teste do qui-quadrado e o teste $T$ de Student. O software estatístico utilizado nas análises foi o MINITAB versão 14.2. Entre os principais resultados, destaca-se que $25,4 \%$ dos restaurantes fazem uso de informação nutricional e/ou de saúde. O tipo de informação nutricional mais empregado é a "declaração de nutrientes", com relevância para a informação do valor energético e de macronutrientes de alguns pratos. A fonte mais citada para a disposição da informação nutricional foi o cardápio, com 48,3\% das citações. A freqüência de oferecimento dessas informações é significativamente maior entre as redes de fast food do que entre os restaurantes full service. Aponta-se a necessidade de se estudar formas de regulamentação dessas informações pelo poder público.
\end{abstract}

Palavras-chave: serviços de alimentação; rotulagem nutricional; legislação; informação ao consumidor.

\begin{abstract}
The main objective of this study was to identify and characterize nutritional and health information made available in commercial restaurants in the city of Campinas, SP, Brazil. Pre-tested questionnaires were used to interview twenty managers of fast food and ninety-four of full service restaurants, between October and December of 2005, located in the five administrative regions of Campinas. After collecting the information, a databank was created using Microsoft Excel software. The chi-square and $t$ Student tests and the statistical software MINITAB version 14.2 were used for the statistical treatment of the data. Among the main results, the fact that $25.4 \%$ of the restaurants provided nutritional and/or health information, stood out. The most frequently provided type of nutritional information was the nutrient declaration highlighting the energy value and macronutrients of some foods. Nearly most of the respondents $(48,3 \%)$ mentioned the use of the menu to display nutritional information. The frequency with which this information was provided was significantly higher in the fast food networks than amongst the full service restaurants. The need for public authorities to study ways of regulating such information was pointed out.

Keywords: catering services; nutritional labeling; legislation; consumer information.
\end{abstract}

\section{Introdução}

Uma tendência da prática alimentar nos últimos anos é o crescimento da freqüência do consumo de refeições realizadas fora do domicílio (STEWART; BLISARD; JOLLIFFE, 2006).

Segundo a Pesquisa de Orçamento Familiar (POF) de 2003-2004, o percentual de despesas com alimentação fora do domicílio entre a população residente em áreas urbanas (25,7\%) é praticamente o dobro daquele observado nas áreas rurais $(13,1 \%)$. Além disso, a Região Sudeste é onde a população mais gasta com alimentação fora do domicílio $(26,9 \%)$ e a maior parte da despesa destina-se ao almoço e ao jantar $(10,1 \%$ do total gasto com alimentos) (IBGE, 2004).

De acordo com a ABIA (2005), dentre os canais operadores do serviço de alimentação, existem os canais comerciais. Estes têm como objetivo principal a obtenção de lucratividade com a comercialização de refeições. Os restaurantes comerciais com- preendem a maior fatia da receita anual dos canais integrantes do serviço de alimentação, o faturamento do setor em 2001 foi de $23,0 \%$, seguido pelas padarias (15\%) e bares $(14,4 \%)$.

Atualmente observam-se iniciativas nos serviços de alimentação em informar a qualidade nutricional das refeições (SALAY, 2001). Um exemplo desse tipo de iniciativa no Brasil é mostrado pela Sociedade Brasileira de Cardiologia/Fundo de Aperfeiçoamento e Pesquisa em Cardiologia - SBC/FUNCOR. A instituição desenvolveu o selo de Aprovação de Qualidade SBC/FUNCOR, instituído em 1991, que tem como parâmetros fundamentais os níveis de gordura e colesterol contidos nos alimentos. O restaurante Biosfera da cidade de São Paulo recebeu o selo de aprovação pela SBC/FUNCOR para quatro pratos (SBC/FUNCOR, 2002).

Recebido para publicação em 4/6/2007

Aceito para publicação em 30/11/2007 (002578)

Departamento de Alimentos e Nutrição, Faculdade de Engenharia de Alimentos, Universidade Estadual de Campinas - Unicamp, Rua Monteiro Lobato, 80, CP 6121,

CEP 13083-862, Campinas - SP, Brasil,E-mail:van.maestro@gmail.com; salay@fea.unicamp.br

${ }^{*}$ A quem a correspondência deve ser enviada 
Todavia, não se conhece a proporção de restaurantes que veiculam algum tipo de informação nutricional e de saúde no Brasil. Este dado, no entanto, pode servir como valioso subsídio para a elaboração de programas envolvendo a informação nutricional e de saúde, incluindo a regulamentação.

Em nível internacional, a trajetória da rotulagem nutricional em restaurantes teve início em 1993, quando a Food and Drug Administration (FDA) editou as regulamentações de acordo com a Nutrition Labeling and Education Act (NLEA) que exigiu dos restaurantes a concordância $\mathrm{e} o$ atendimento às regulamentações elaboradas sobre as informações nutricionais e as alegações de saúde que aparecessem em quadros ou placas de anúncio. Em 1996, ocorreu a regulamentação que os restaurantes devem seguir para o oferecimento de informação nutricional e de saúde sobre os itens listados no cardápio. Cabe esclarecer que a FDA estimula, mas não obriga, a disponibilidade dessas informações nos alimentos comercializados pelos restaurantes (KURTZWEIL, 1997).

Segundo o Code of Federal Regulations (2002), a "rotulagem nutricional" é um termo que pode ser empregado em restaurantes para se referir às informações nutricionais e deve estar disponível de forma clara no ponto de compra através de cardápios, apostilas, folders, entre outros veículos.

Com base nas regulamentações da FDA, a National Restaurant Association (NRA) possui vários estabelecimentos alimentícios associados, como por exemplo: Burger King, McDonald's, Sodexho, Subway e KFC que fornecem informações relativas ao conteúdo energético e de nutrientes dos itens do cardápio (NRA, 2006). A European Modern Restaurant Association (EMRA) também possui vários restaurantes associados fornecendo informação calórica e nutricional de seus produtos como a Pizza Hut na França e o KFC na Alemanha. Porém, em meados da presente década, é que se iniciou o processo de regulamentação dessas informações, tendo como referencial dados da British Food Standards Agency's (EMRA, 2004).

De acordo com Weimer (1999), os programas de informação nutricional objetivam aumentar o conhecimento do consumidor sobre nutrição, de modo a instruí-lo na busca por dietas mais benéficas à saúde.

Sloan e Bell (1999) e Kurtzweil (1997) alegam que a rotulagem nutricional em restaurantes, por meio das informações nutricionais e de saúde nos cardápios, pode ajudar o consumidor a entender melhor o papel da dieta para a promoção da saúde e a efetuar escolhas de alimentos que contribuam para uma refeição saudável.

A presente pesquisa teve como objetivos conhecer as iniciativas de oferecimento de informação nutricional e de saúde em restaurantes comerciais do tipo fast food e full service do município de Campinas-SP. Serão identificados os tipos de informação e de veículos de disseminação. Procurou-se, por meio de testes estatísticos, identificar a associação entre variáveis que representam as características dos estabelecimentos e aquelas relativas ao provimento das informações.

\section{Material e métodos}

A população de estudo foi composta por gerentes de serviços de alimentação comerciais da cidade de Campinas - SP. Estes estabelecimentos foram divididos em serviços rápidos, de conveniência (fast food) e serviços completos (de mesa), em geral com cardápios mais variados (full service), conforme classificação empregada pelo U.S. Department of Commerce Economics and Statistics Administration (2000).

Dados do Instituto Brasileiro de Geografia e Estatística - IBGE (2005) mostram que Campinas possuía, na época do levantamento das informações sobre o município, uma população estimada em 1.045.706 habitantes. Segundo a ACIC (2004), o tamanho de Campinas representa 42,0\% do PIB da Região Metropolitana de Campinas (RMC), que gerou bens e serviços na ordem de R \$ 78,2 bilhões em 2004. Segundo a Target Marketing (2006), Campinas alcançou o nono lugar no Índice da Potencialidade de Consumo (IPC), entre os 500 maiores municípios estudados.

Devido à inexistência de um cadastro único e atualizado dos restaurantes do município de Campinas, obteve-se a listagem dos serviços de alimentação comerciais através de consultas em guias do município disponíveis em páginas da internet: Listão Oesp (2004), Prefeitura Municipal de Campinas (2005), Portal dos Restaurantes de Campinas (2005), CEASA (2005), Guia EPTV (2004) e sítios eletrônicos específicos das grandes redes de fast food, além de consulta em listas telefônicas. Esta pesquisa resultou num conjunto de 107 restaurantes fast food e 507 restaurantes full service listados. Contatos por telefone foram realizados para a confirmação do tipo de linha de atuação do serviço de alimentação e endereço completo.

Para a realização do dimensionamento da amostra, primeiramente foi definida uma probabilidade de confiança de $95 \%$ (erro amostral de 5\%), utilizando-se a metodologia proposta por Cochran (1977).

Em seguida, definiu-se o sorteio aleatório entre os restaurantes listados, tendo por base as 5 regiões (Leste, Norte, Sul, Noroeste e Sudoeste) de Campinas. Tal procedimento permitiu a obtenção de amostragem aleatória estratificada, totalizando 114 restaurantes, sendo 20 do tipo fast food e 94 do tipo full service.

A coleta de dados foi realizada por meio de entrevista com os gerentes dos restaurantes, no período de outubro a dezembro de 2005, com a aplicação de um questionário contendo questões abertas, semi-estruturadas e estruturadas e que foi pré-testado. O pré-teste foi realizado em setembro de 2005 e possibilitou o aprimoramento do instrumento.

O pré-teste serve para avaliar, segundo Mattar (2005), o tempo despendido com a entrevista, a compreensão das questões pelos respondentes, a necessidade de se completar as opções das questões fechadas, entre outros objetivos.

Separadamente do questionário, foi elaborado um material explicativo sobre a elaboração de informação nutricional e de saúde em restaurantes para auxiliar os gerentes a responderem às questões da entrevista. 
O referido material baseou-se nas informações sobre rotulagem de alimentos da ANVISA (1999) e ANVISA (2003). Segundo esse órgão, as declarações podem ser de dois tipos: a "declaração de nutrientes" (informação nutricional que se refere à declaração do conteúdo de energia e/ou nutrientes de um alimento); e a "declaração de propriedades nutricionais" (refere-se à declaração de propriedades nutricionais particulares do alimento: livre, light, fonte de, etc.). Pode ser também utilizados dois tipos de alegações: a "alegação de propriedade funcional e/ou a alegação de propriedade de saúde" (refere-se ao papel metabólico ou fisiológico do nutriente para as funções normais do organismo humano e/ou sugere a existência da relação entre o alimento ou ingrediente com doença ou condição relacionada à saúde).

O levantamento de dados foi realizado de forma criteriosa pela primeira autora deste artigo e por três alunos do Grupo de Estudos e Projetos em Engenharia de Alimentos (GEPEA) da Universidade Estadual de Campinas, devidamente treinados. A capacitação da equipe também contou com o uso de um manual do entrevistador elaborado especificamente para a pesquisa.

Após a aplicação do questionário, foi construído um banco de dados utilizando o software Excel (2000). Para o tratamento estatístico, utilizou-se o teste do qui-quadrado e o teste $T$ de Student.

O software estatístico utilizado nas análises foi o MINITAB System (MINITAB Institute Inc., 2005).

\section{Resultados e discussão}

Na Tabela 1, estão descritos o perfil dos gerentes integrantes da amostra e algumas características dos restaurantes comerciais. Quase a metade $(48,3 \%)$ do total de gerentes entrevistados apresenta escolaridade até o segundo grau completo e apenas 2,6\% concluíram cursos de pós-graduação. Entre os entrevistados, aproximadamente $60 \%$ possuem curso na área de ciências exatas e engenharias. É interessante frisar que somente $20,3 \%$ dos gerentes concluíram cursos relacionados à área de nutrição ou alimentos e bebidas. Quanto aos estabelecimentos, a maioria classifica-se como microempresa $(63,2 \%)$, que atuam em full service (82,5\%), na região leste de Campinas (69,3\%) e com forma jurídica de sociedade $(47,4 \%)$.

Nota-se que a média dos números de refeições servidas por dia nos restaurantes com linha de atuação fast food foi significativamente superior à média do número de refeições servidas pela linha de atuação full service (teste $T$ de Student; $p=0,013$ ).

Na Tabela 2, verifica-se que apenas 29 das 114 empresas disponibilizam informação nutricional e/ou de saúde no serviço de alimentação, correspondendo a $25,4 \%$ da amostra. Desses 29 restaurantes, 14 são do tipo fast food e 15 do tipo full service. Cabe frisar que do total de restaurantes que não fornecem essas informações, apenas $7,1 \%$ são do tipo fast food, enquanto $92,9 \%$ são do tipo full service.

Foi captada associação significativa entre o oferecimento de informação nutricional e/ou de saúde e a linha de atuação. Percebe-se que a freqüência de oferecimento dessas informações é maior entre as redes de fast food do que entre os restaurantes full service.

Inversamente, não foi observada associação significativa entre o oferecimento de informação nutricional e/ou de saúde e o tipo de estrutura do estabelecimento (qui-quadrado; $p=0,201)$.

Dos 29 restaurantes da pesquisa que oferecem informação nutricional e/ou de saúde, 15 estabelecimentos utilizam a "declaração de nutrientes"; 19, a "declaração de propriedades nutricionais"; e, apenas dois, a "alegação de propriedade funcional e/ou de saúde", correspondendo a 51,7, 65,5 e 6,9\% dos valores, respectivamente.

Nas Tabelas 3, 4 e 5, a seguir, está especificado cada um dos tipos de declaração e alegação citadas.

Os dados da Tabela 3 revelam que, entre os gerentes de restaurantes que citaram disponibilizar a "declaração de nutrientes", a maioria deles oferece informação nutricional referente ao valor energético dos alimentos e de seus macronutrientes (proteínas, carboidratos e gorduras). Os minerais e as vitaminas apresentaram, em geral, apenas uma citação em relação ao atributo "fonte de" e nenhuma citação para o atributo "alto teor".

Em relação aos tipos de "declaração de propriedades nutricionais", observa-se, na Tabela 3, que o atributo light recebeu o maior número de citações (21) quando comparado aos demais. Registra-se também que, dentre estas citações, 15 (78,9\%) pertencem às declarações de redução do valor energético dos pratos, seguido pelas gorduras totais, com quatro citações, correspondendo a $21,1 \%$. Em relação ao atributo livre, o nutriente mais citado foi o açúcar, com cinco citações (26,3\%), representando os alimentos livres de açúcares de adição, ou seja, as sobremesas diet.

O percentual de citações $(5,3 \%)$ foi igual para o conteúdo de colesterol, gordura saturada, fibras, vitaminas e sais minerais, independentemente dos respectivos atributos (livre, light, fonte de ou alto teor). Observa-se, também, que o referido percentual foi muito inferior quando comparado aos valores apontados no parágrafo acima.

De acordo com a Tabela 4, podemos afirmar que a "alegação de propriedade funcional e/ou de saúde" está presente em menor proporção nos restaurantes, sendo a fibra alimentar o atributo mais citado.

Um exemplo de "alegação de propriedade de saúde" aplicado em um restaurante da pesquisa é: "As fibras auxiliam contra a constipação intestinal".

Com relação ao tipo de cálculo nutricional, 10 dos 29 gerentes entrevistados responderam que as informações são oriundas de análise bromatológica realizada por laboratórios de análise de alimentos, 7 obtêm esses dados com o uso de tabelas de composição química de alimentos e 12 responderam que não há cálculo nutricional.

A ausência de cálculo nutricional para 11 das 12 empresas foi motivada pelos seguintes fatores: prato composto por carnes, aves, peixes, embutidos ou queijos naturalmente magros ou light; prato composto por carne, ave ou peixe grelhado/cozido/ 
Tabela 1. Perfil dos gerentes entrevistados e caracterização dos restaurantes. Campinas-SP, 2005.

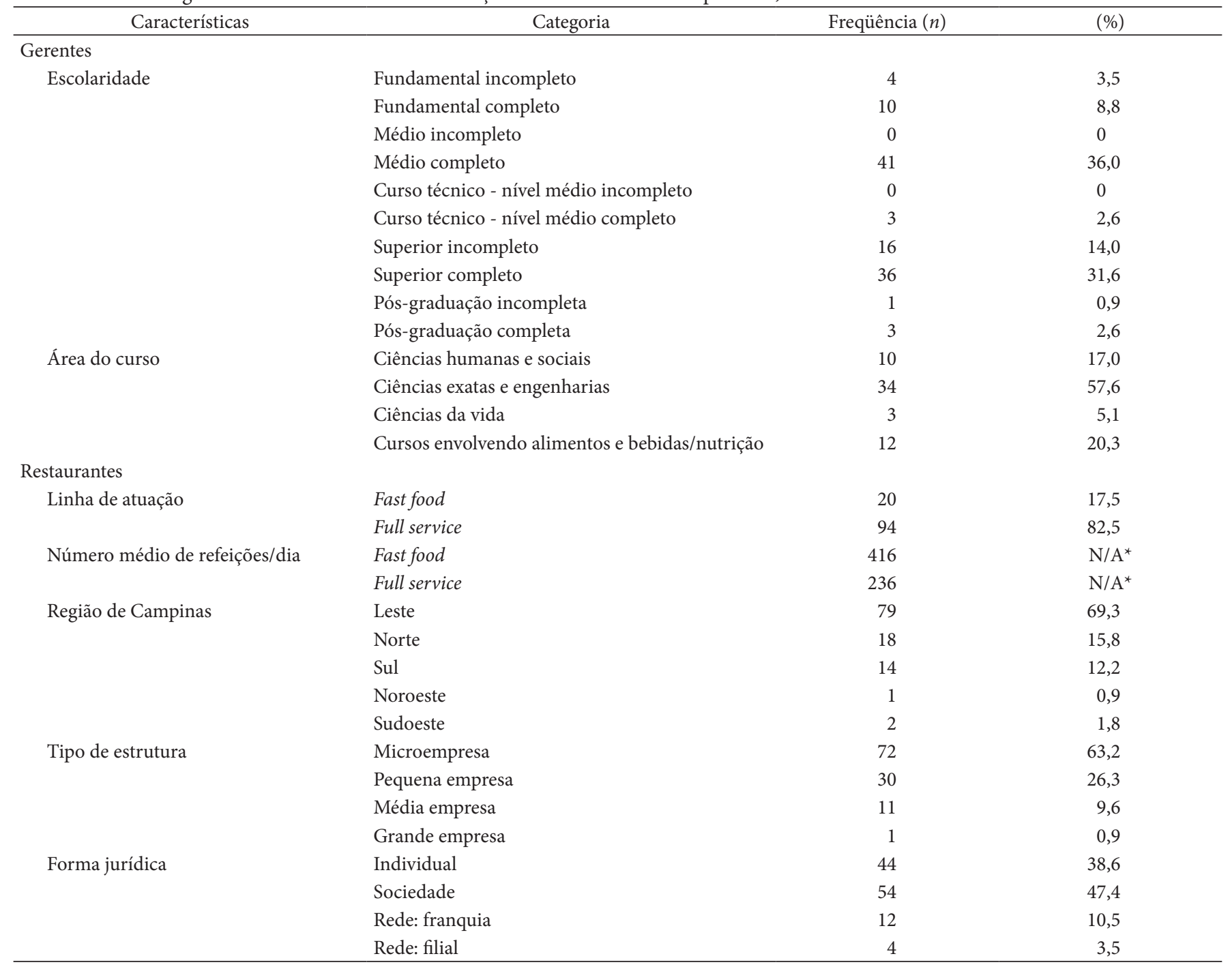

${ }^{*}$ N/A: não aplicável.

Tabela 2. Disponibilidade de informação nutricional e/ou de saúde de acordo com a linha de atuação do estabelecimento. Campinas-SP, 2005.

\begin{tabular}{cccc}
\hline Linha de atuação & \multicolumn{3}{c}{ Disponibilidade } \\
\cline { 2 - 4 } & Sim & Não & Total \\
\hline Fast Food & 14 & 06 & $20[17,5]$ \\
& $(48,3)$ & $(7,1)$ & \\
Full Service & 15 & 79 & $94[82,5]$ \\
& $(51,7)$ & $(92,9)$ & \\
Total & $29[25,4]$ & $85[74,6]$ & $114[100,0]$ \\
\hline
\end{tabular}

$\chi^{2}=\mathrm{p}<0,001$, significativo a 5\%; Nota: os números entre colchetes são os percentuais em relação ao número de restaurantes $(n=114)$ que compõem a amostra; e os números entre parênteses são os percentuais em relação ao total observado em cada coluna.

assado; prato composto por hortaliças folhosas e/ou legumes. Duas empresas relataram sobremesas isentas de açúcares adicionados e uma empresa relatou sobremesas compostas por ingredientes light.

Apenas 6 das 29 empresas que disponibilizam informação nutricional e/ou de saúde oferecem também a adequação nu- tricional o que corresponde a 20,7\%. Uma delas baseia-se em valores diários de referência para adultos e crianças e quatro, somente para adultos. A adequação nutricional nos restaurantes foi feita tanto para as opções de pratos como também para produtos alimentícios como salgados, sobremesas, bebidas. A sexta empresa ofereceu adequação nutricional com base na pirâmide alimentar.

Na Tabela 5, verifica-se que a fonte mais citada para a disponibilidade da informação nutricional e/ou de saúde foi o cardápio, com 48,3\% das citações, seguido pela página eletrônica e apostila, ambos com $20,7 \%$ das citações.

\subsection{Discussão}

O tipo de informação nutricional mais empregado nos restaurantes comerciais estudados é a "declaração de nutrientes", com destaque para a informação do valor energético e de macronutrientes de alguns pratos. 
Tabela 3. Distribuição dos tipos de "declaração de nutrientes" e "declaração de propriedades nutricionais" disponibilizadas por restaurantes comerciais. Campinas-SP, 2005.

\begin{tabular}{|c|c|c|c|c|}
\hline \multicolumn{5}{|c|}{ Tipos de "declaração de nutrientes" } \\
\hline Atributos & \multicolumn{2}{|c|}{$n$} & \multicolumn{2}{|c|}{$\%^{*}$} \\
\hline Valor energético & \multicolumn{2}{|c|}{14} & \multicolumn{2}{|c|}{93,3} \\
\hline Proteínas & \multicolumn{2}{|c|}{11} & \multicolumn{2}{|c|}{73,3} \\
\hline Gorduras totais & \multicolumn{2}{|c|}{10} & \multicolumn{2}{|c|}{66,7} \\
\hline Gorduras saturadas & \multicolumn{2}{|c|}{8} & \multicolumn{2}{|c|}{53,3} \\
\hline Colesterol & \multicolumn{2}{|c|}{7} & \multicolumn{2}{|c|}{46,7} \\
\hline Fibra alimentar & \multicolumn{2}{|c|}{8} & \multicolumn{2}{|c|}{53,3} \\
\hline Cálcio & \multicolumn{2}{|c|}{6} & \multicolumn{2}{|c|}{40,0} \\
\hline Ferro & \multicolumn{2}{|c|}{5} & \multicolumn{2}{|c|}{33,3} \\
\hline Sódio & \multicolumn{2}{|c|}{6} & \multicolumn{2}{|c|}{40,0} \\
\hline Outros: Zinco & \multicolumn{2}{|c|}{1} & \multicolumn{2}{|c|}{6,7} \\
\hline \multicolumn{5}{|c|}{ Tipos de "declaração de propriedades nutricionais" } \\
\hline \multirow[t]{2}{*}{ Atributos } & \multicolumn{2}{|c|}{ Livre $^{\star \star}$} & \multicolumn{2}{|c|}{ Light $^{* * *}$} \\
\hline & $n$ & $\%^{*}$ & $n$ & $\%^{*}$ \\
\hline Valor energético & 1 & 5,3 & 15 & 78,9 \\
\hline Gorduras totais & 1 & 5,3 & 4 & 21,1 \\
\hline Gorduras saturadas & 0 & 0 & 1 & 5,3 \\
\hline Colesterol & 0 & 0 & 1 & 5,3 \\
\hline \multirow[t]{2}{*}{ Açúcares } & 5 & 26,3 & 0 & 0 \\
\hline & \multicolumn{2}{|c|}{ Fonte $\mathrm{de}^{\star * * *}$} & \multicolumn{2}{|c|}{ Alto teor ${ }^{* * * * *}$} \\
\hline Fibra alimentar & 1 & 5,3 & 0 & 0 \\
\hline Cálcio & 1 & 5,3 & 0 & 0 \\
\hline Ferro & 1 & 5,3 & 0 & 0 \\
\hline Outros: Potássio & 1 & 5,3 & 0 & 0 \\
\hline Vitamina A & 1 & 5,3 & 0 & 0 \\
\hline Vitamina C & 1 & 5,3 & 0 & 0 \\
\hline
\end{tabular}

*Nota: os valores percentuais correspondem ao número de gerentes $(n=15)$ e $(n=19)$ que citaram a "declaração de nutrientes" e a "declaração de propriedades nutricionais", respec-

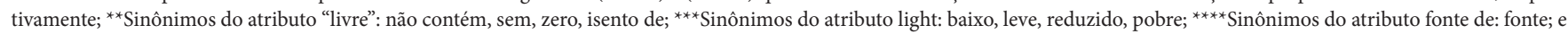

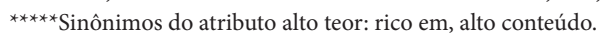

Tabela 4. Distribuição dos tipos de "alegações de propriedade funcional e/ou de saúde” disponibilizadas pelos restaurantes a informação nutricional. Campinas-SP, 2005.

\begin{tabular}{llr}
\hline \multicolumn{1}{c}{ Atributos } & $n$ & $\%^{*}$ \\
\hline Ácidos graxos ômega & 1 & 50,0 \\
Licopeno & 1 & 50,0 \\
Fibra alimentar & 2 & 100,0 \\
Proteína de soja & 1 & 50,0 \\
Outros: Flavonóides & 1 & 50,0 \\
\hline
\end{tabular}

${ }^{*}$ Nota: os valores percentuais correspondem ao número de gerentes $(n=2)$ que citaram a "alegação de propriedade funcional e/ou de saúde".

Uma possível explicação para este fato é que os gerentes captaram o interesse dos comensais em conhecer o valor energético, de gorduras totais e de açúcares das refeições. Na verdade, estes elementos têm sido intensamente tratados por diversas fontes de informação como a mídia e as propagandas de alimentos.

Por outro lado, supõe-se, também, que valores referentes a certos componentes, como o conteúdo de colesterol e gordura saturada possam ser informações de mais difícil entendimento pelos consumidores.

Uma pesquisa conduzida pelo National Heart, Lung, and Blood Institute - NHLBI, de 1982 a 1995, a respeito do conhecimento dos consumidores sobre os fatores dietéticos e sua relação
Tabela 5. Veículos utilizados por restaurantes comerciais para disponibilizar informação nutricional e/ou de saúde. Campinas-SP, 2005.

\begin{tabular}{lcc}
\hline \multicolumn{1}{c}{ Veículos } & $n$ & $\%^{*}$ \\
\hline Cardápio & 14 & 48,3 \\
Cartazes & 2 & 6,9 \\
Quadros & 2 & 6,9 \\
Folders & 3 & 10,3 \\
Anúncios em jornal & 0 & 0 \\
SAC (Serviço de Atendimento & 0 & 0 \\
ao Consumidor) - Ligação & & \\
gratuita (0800) & & \\
Página eletrônica & 6 & 20,7 \\
Banner & 2 & 6,9 \\
Apostila & 6 & 20,7 \\
Placas & 1 & 3,4 \\
Display de balcão & 2 & 6,9 \\
Expositor de fotos & 1 & 3,4 \\
Lâmina de bandeja & 1 & 3,4 \\
Embalagem do produto & 1 & 3,4 \\
\hline
\end{tabular}

${ }^{*}$ Nota: os valores percentuais estão relacionados com o número de restaurantes $(n=29)$ que oferecem informação nutricional e/ou de saúde.

com doenças cardíacas, foi analisada e comentada por Guthrie, Derby e Levy (1999). De acordo com estes autores, a referida pesquisa revelou que poucos consumidores mencionaram a 
gordura saturada como um importante fator de risco da dieta e, segundo os mesmos autores, a interpretação para isto é a tentativa dos indivíduos de simplificar a "norma dietética", ou seja, o público pode estar menos interessado em informações nutricionais mais detalhadas, pois isso complica sua tarefa na seleção de alimentos.

Uma pesquisa realizada na Pensilvânia (EUA) envolveu 150 consumidores de um restaurante universitário e teve como objetivos disponibilizar informações nutricionais em alguns pratos quentes de entrada e verificar sua influência no comportamento dos indivíduos durante a escolha das refeições (CRANAGE; CONKLIN; LAMBERT, 2004). As informações nutricionais, por porção, consideradas mais importantes e, portanto, mais lidas entre os consumidores dizem respeito ao valor energético, gorduras totais e kilocalorias provenientes das gorduras em detrimento dos valores de colesterol, sódio, carboidratos totais e proteínas.

De acordo com os referidos autores, os pratos quentes de entrada (torta de queijo com espinafre, quiche de brócolis com queijo, rolo primavera vegetariano, etc.) com conteúdo maior de gordura foram escolhidos com menor freqüência e viceversa, quando a informação nutricional foi disponibilizada aos consumidores, o que não ocorria em situação anterior, a qual se caracterizava pela inexistência de informação nutricional no restaurante. Outro aspecto importante foi que o oferecimento desse tipo de informação ampliou o consumo de legumes e verduras e reduziu o consumo de batatas fritas e de sobremesas entre os consumidores.

O tipo de "alegação de propriedade funcional e/ou de saúde" mais citado pelos gerentes de restaurantes foi relativo à fibra alimentar. Na verdade, houve um aumento da disponibilidade e das propagandas de produtos alimentícios com alto teor de fibras no Brasil nas décadas recentes.

A existência de informação nutricional foi maior nos restaurantes fast food. Provavelmente o ocorrido se deva ao fato das franquias possuírem uma política facilitadora desse tipo de iniciativa, pois, geralmente, estabelecem convênios com laboratórios de análise nutricional e o custo é cotizado entre as empresas. Outro fator importante é que a maioria das franquias de fast food possui um responsável técnico que pode elaborar os cálculos nutricionais usando as tabelas de composição química dos alimentos, sem custo adicional para a empresa.

Cabe ainda ressaltar a pressão social em países cuja população possui maiores rendimentos sobre as cadeias de fast food para a produção de alimentos mais saudáveis e sua divulgação ao consumidor. Em Nova York, por exemplo, várias dessas cadeias têm processos arquivados alegando que os alimentos industrializados de baixo valor nutricional têm contribuído para a existência de diabetes, doenças cardiovasculares e a obesidade (COESTIER; GOZLAN; MARETTE, 2005).

Entre os veículos de informação nutricional e/ou de saúde, o cardápio revelou-se como o mais freqüente (14 citações), seguido pela apostila (disponibilizada ao cliente somente quando solicitada) e pela página da internet, ambas com seis citações. A disposição da informação no cardápio, à vista do consumidor, atua como uma estratégia de marketing. $\mathrm{O}$ uso de página ele- trônica, escolhida pelos gerentes como veículo de informação externa ao restaurante, com vistas ao fornecimento do valor nutricional das preparações comercializadas no estabelecimento, justifica-se pelo fato de a internet viabilizar a comunicação entre os serviços oferecidos pela empresa e o consumidor.

Uma pesquisa de Sneed e Burkhalter (1991), que teve por base entrevista com 70 dos 400 diretores de pesquisa e desenvolvimento de grandes corporações de serviços de alimentação listadas no periódico Restaurants \& Institutions de 1993, apontou que das 27 empresas que ofereciam informação nutricional, apenas duas utilizavam o cardápio para disseminar a informação, ao passo que 15 companhias usavam uma apostila que era mostrada ao consumidor de acordo com sua solicitação.

O uso de apostilas pode não ser a opção mais adequada para informar sobre nutrição aos consumidores, pois não é uma alternativa prontamente acessível. Almanza e Hsieh (1995), em entrevista com 86 indivíduos, alunos e funcionários de um restaurante universitário em Purdue, diagnosticaram que depois do atributo atratividade, a facilidade de uso da informação nutricional foi um dos segundos maiores atributos que serviam de estímulo aos consumidores para se informarem sobre nutrição.

Destaca-se, nesta pesquisa, que apenas $29(25,4 \%)$ dos 114 restaurantes da amostra disponibilizam informação nutricional e/ou de saúde em seus estabelecimentos. Estudo conduzido nos Estados Unidos por meio de entrevista com 65 diretores de pesquisa e desenvolvimento de grandes corporações de serviços de alimentação, diagnosticou que somente 23 deles $(35,4 \%)$ disponibilizavam informação nutricional nos restaurantes (ALMANZA; NELSON; CHAI, 1997).

Salay (2003) afirma que o consumidor está pouco preocupado com alguns atributos nutricionais dos alimentos. Conseqüentemente, as empresas terão pouco estímulo para oferecer essas informações.

Os dados levantados apontam a necessidade de se elaborar algum tipo de regulamentação em relação ao oferecimento dessas informações e que sejam coerentes com a realidade socioeconômica do setor.

Quase a metade dos restaurantes que oferece informação nutricional não realiza nenhum tipo de cálculo de nutrientes em relação à alegação "declaração de propriedades nutricionais", principalmente em relação ao atributo light em calorias devido à utilização de ingredientes com teor de gordura reduzido como, por exemplo, os queijos brancos, ausência de fritura, presença de saladas ou uso de produtos industrializados light. Esse tipo de procedimento aplicado nos pratos dos restaurantes é aleatório. O correto é realizar o cálculo energético de um prato convencional, informando sua composição e usá-lo como padrão de comparação com os demais pratos light, apontando claramente uma redução de no mínimo $25 \%$ das calorias desse prato em relação ao outro.

A “declaração de propriedades nutricionais" é uma das mais complexas em termos de análise nutricional, pois para cada nutriente (proteína, gordura, ferro, vitamina A, entre outros) existem vários atributos (baixo, não contém, etc.) e cada um 
deles com um referencial diferente do teor de nutrientes para o cálculo.

Outra preocupação relevante que pode gerar muita confusão é atribuir ao alimento uma declaração de propriedade funcional e/ou de saúde que ele não tem, como por exemplo, o auxílio na cura ou prevenção de doenças. É devido a essa preocupação que a ANVISA (2005) estabelece uma lista dos alimentos aprovados que apresentam alegação de propriedade funcional e/ou de saúde que pode ser colocada nos rótulos dos alimentos e a forma como essa propriedade pode ser citada.

Vale também chamar a atenção para o pequeno percentual de restaurantes $(20,7 \%)$ que disponibiliza a adequação nutricional dos produtos oferecidos.

Um levantamento do Instituto Brasileiro de Defesa do Consumidor (IDEC) verificou que várias redes de fast food não informam a adequação nutricional de seus produtos, comercializando sanduíches com conteúdo de gordura e sal que se aproximam da quantidade diária total recomendada pela Organização Mundial de Saúde (IDEC, 2005).

Atualmente, algumas iniciativas em âmbito nacional, estadual ou municipal estão emergindo a respeito de políticas que regulamentam e fiscalizam o uso responsável de informação nutricional nas embalagens dos alimentos vendidos em redes de fast food.

A ANVISA, por exemplo, é incumbida de regulamentar e fiscalizar a rotulagem nutricional dos alimentos preparados e embalados em restaurantes e estabelecimentos comerciais, como sanduíches e sobremesas, somente no caso de serem comercializados para outros estabelecimentos.

O IDEC está solicitando que as redes de fast food do País disponham informações nutricionais nas embalagens dos alimentos. Segundo o Instituto, a não adoção dessas informações pelas empresas implica no recebimento de uma notificação devido ao descumprimento da resolução n ${ }^{\circ} 360$ de 2003 da ANVISA e do Código de Defesa do Consumidor (IDEC, 2006).

Em âmbito municipal, a prefeitura do Rio de Janeiro editou um decreto $\mathrm{n}^{\circ} 23.148$ de 17/07/03 estabelecendo que as redes de fast food adotem uma tabela legível e visível ao público contendo a informação nutricional de todos os produtos oferecidos com base na legislação vigente para rotulagem nutricional de produtos industrializados. O órgão responsável pela fiscalização e notificação é a Vigilância Sanitária do município (Vigilância Sanitária do Município do Rio de Janeiro, 2003).

Em âmbito nacional, também estão surgindo iniciativas de políticas de saúde dirigidas aos serviços de alimentação das escolas. O Ministro de Estado da Saúde e o Ministro de Estado da Educação instituíram a portaria no 1.010 de 08/05/06 cujas diretrizes visam à promoção da alimentação saudável nas escolas de educação infantil, fundamental e nível médio das redes públicas e privadas. A referida portaria restringe, por exemplo, a oferta e a venda de alimentos com alto teor de gordura, açúcar e sal e estimula o aumento da oferta e do consumo de frutas, legumes e verduras (MINISTÉRIO DA SAÚDE, 2006).

\section{Conclusões}

O tipo de informação nutricional mais disponibilizado nos restaurantes refere-se à "declaração de nutrientes", principalmente em relação ao conteúdo energético e de macronutrientes das preparações. O cardápio foi o veículo mais citado para disponibilizar essas informações.

A informação nutricional é oferecida com maior freqüência nos restaurantes do tipo fast food. Observa-se que a política empresarial desse tipo de estabelecimento contribui para o ocorrido, enquanto os restaurantes do tipo full service não possuem nenhuma estratégia facilitadora e estimulante para esse tipo de medida.

No município de Campinas, com base na amostra estudada, nota-se que é pequena a disponibilidade de informação sobre o valor nutricional dos alimentos oferecidos ao consumidor em restaurantes e que existem estabelecimentos que não realizam cálculo nutricional para o atributo light.

Os serviços de alimentação devem apresentar informações nutricionais que tenham fundamento científico, a fim de não confundir os consumidores, informando-os do conteúdo nutricional de alimentos que podem prejudicar ou beneficiar a sua saúde. Deste modo, faz-se urgente a necessidade de se elaborarem critérios de regulamentação e fiscalização dessas informações pelo poder público.

Ressalta-se que será um desafio muito grande, principalmente para os restaurantes do tipo full service, oferecer, de forma criteriosa, produtos diferenciados no teor de nutrientes como uma sobremesa sem adição de açúcares ou um prato light em calorias, por exemplo, pois isso envolve cálculos nutricionais fidedignos, que seguramente demandam custos.

As diferenças entre os restaurantes, tais como o tipo, tamanho, estilo de operação, volume de venda anual, entre outras, torna a padronização do oferecimento de informação nutricional e de saúde uma tarefa difícil.

Porém, a presente pesquisa aponta o surgimento de algumas iniciativas individuais, de alguns proprietários de restaurantes, em oferecer informação nutricional, gerando a necessidade de apoio governamental, para que um número cada vez maior de estabelecimentos seja estimulado a adotar essa medida.

A maior preocupação com a nutrição está levando o cliente a demandar alternativas saudáveis em restaurantes, e o presente estudo aponta que estes locais podem ser um importante foco para programas de informação nutricional, evidenciando uma nova área de atuação no âmbito da saúde pública, com o intuito de melhorar a qualidade de vida da população.

Diante dessa realidade, faz-se necessária a criação de políticas públicas facilitadoras, como o oferecimento de cursos e treinamentos aos funcionários dos restaurantes, que estimulem a disposição de informações nutricionais claras e confiáveis aos consumidores a fim de que estes tenham elementos para escolhas mais saudáveis. 


\section{Agradecimentos}

À FAEPEX (UNICAMP) e à CAPES, pelo apoio financeiro à pesquisa; e à empresa EasyStat, pela elaboração de todas as análises estatísticas que integram o estudo.

\section{Referências bibliográficas}

ALMANZA, B. A.; HSIEH, H. M. Consumer preferences among nutrition labeling formats in a restaurant. Journal of the American Dietetic Association, v. 95, n. 1, p. 83-85, 1995.

ALMANZA, B. A.; NELSON, D.; CHAI, S. Obstacles to nutrition labeling in restaurants. Journal of the American Dietetic Association, v. 97, n. 2, p. 157-161, 1997.

ABIA - ASSOCIAÇÃO BRASILEIRA DAS INDÚSTRIAS DA ALIMENTAÇÃO. A nova distribuição para o food service brasileiro. 2005. Disponível em: http://www.abia.org.br/noticias. asp

ACIC - ASSOCIAÇÃO COMERCIAL E INDUSTRIAL DE CAMPINAS. Relatório Econômico 2004. Disponível em: <http:// www.acicnet.org.br>. Acesso em: 18 jul. 2006.

BRASIL. AGÊNCIA NACIONAL DE VIGILÂNCIA SANITÁRIA ANVISA. Alimentos com alegações de propriedades funcionais e ou de saúde, novos alimentos/ingredientes, substâncias bioativas e probióticos. Lista das alegações aprovadas. Disponível em: $<$ http:// www.anvisa.gov.br>. Acesso em: 25 ago. 2005.

BRASIL. AGÊNCIA NACIONAL DE VIGILÂNCIA SANITÁRIA - ANVISA. Resolução n ${ }^{\circ}$, de 30 de abril de 1999. Diretrizes básicas para análise e comprovação de propriedades funcionais e ou de saúde alegadas em rotulagem de alimentos. Disponível em: <http://www.anvisa.gov.br>. Acesso em: 25 ago. 2005.

BRASIL. AGÊNCIA NACIONAL DE VIGILÂNCIA SANITÁRIA - ANVISA. Resolução RDC n 360, de 23 de dezembro de 2003. Regulamento técnico sobre rotulagem nutricional de alimentos embalados, tornando obrigatória a rotulagem nutricional. Disponível em: <http://www.anvisa.gov.br>. Acesso em: 25 ago. 2005.

BRASIL. Ministério da Saúde. Portaria Interministerial $n^{\circ} 1.010$, de 8 de maio de 2006. Diretrizes para a promoção da alimentação saudável nas escolas de educação infantil, fundamental e nível médio das redes públicas e privadas, em âmbito nacional. Disponível em: <http://www.saude.gov.br>. Acesso em: 19 jun. 2006.

CEASA - Central de Abastecimento de Campinas AS. Restaurantes. Disponível em: <http://www.ceasacampinas.com.br>. Acesso em: 20 jun. 2005.

COCHRAN, W. G. Sampling Techniques. 3 ed. New York: J. Wiley, 1977.

CODE OF FEDERAL REGULATIONS. Nutrition labeling of food. 21 CFR § 101.9. Disponível em: <http://www.gpoaccess.gov>. Acesso em: 11 abr. 2005.

COESTIER, B.; GOZLAN, E.; MARETTE, S. On food companies liability for obesity. American Agricultural Economics Association, v. 87, n. 1, p. 1-14, 2005.

CRANAGE, D. A.; CONKLIN, M. T.; LAMBERT, C. U. Effect of nutrition information in perceptions of food quality, consumption behavior and purchase intentions. Journal of Foodservice Business Research, v. 7, n. 1, p. 43-61, 2004.

EMRA - EUROPEAN MODERN RESTAURANT ASSOCIATION. Roundtable on obesity. Disponível em: <http://www. emrarestaurants.com>. Acesso em: 22 set. 2005.
Guia EPTV. Restaurantes. Disponível em: <http://www.eptv.globo. com/guia2004>. Acesso em: 21 jun. 2005.

GUTHRIE, J. F.; DERBY, B. M.; LEVY, A. S. What people know and do not know about nutrition. In: FRAZAO, E. (Ed.). America's Eating Habits: changes and consequences. Washington, D.C.: United States Department of Agriculture - USDA/Economic Research Service - ERS, 1999. Cap. 13, p. 243-280. (Agriculture Information Bulletin, n. 750).

IDEC - INSTITUTO BRASILEIRO DE DEFESA DO CONSUMIDOR. Embalagem leva Idec a notificar redes de fast food. Disponível em: <http://www.idec.org.br>. Acesso em: 22 set. 2006.

IDEC - INSTITUTO BRASILEIRO DE DEFESA DO CONSUMIDOR. Sal, gorduras e fast food. Disponível em: $<$ http://www.idec.org. br>. Acesso em: 22 set. 2006.

IBGE - INSTITUTO BRASILEIRO DE GEOGRAFIA E ESTATÍSTICA. Cidades@: o Brasil município por município. Disponível em: $<$ http://www.ibge.gov.br>. Acesso em: 18 jul. 2006.

IBGE - INSTITUTO BRASILEIRO DE GEOGRAFIA E ESTATÍSTICA. Pesquisa de orçamentos familiares 2002/2003: primeiros resultados - Brasil e grandes regiões, 2004. Disponível em: $<$ http:// www.ibge.gov.br>.

KURTZWEIL, P. Center for Food Safety and Applied Nutrition. Today's special: nutrition information. Disponível em: $<$ http://www.cfsan. fda.gov>. Acesso em: 15 abr. 2004.

Listão Oesp. Restaurantes. Disponível em: <http://www.listao.com. br>. Acesso em: 16 jun. 2005.

MATTAR, N. M. Pesquisa de Marketing: Metodologia - Planejamento. 6 ed. São Paulo: Atlas, 2005.

NRA - NATIONAL RESTAURANT ASSOCIATION. State menu labeling legislation. Disponível em: $\langle$ http://www.restaurant.org $>$. Acesso em: 22 set. 2006.

Portal dos Restaurantes. Restaurantes. Disponível em: <http://www. portaldosrestaurantes.com.br>. Acesso em: 20 jun. 2005.

Prefeitura Municipal de Campinas. Restaurantes. Disponível em: <http://www.campinas.sp.gov.br>. Acesso em: 16 jun. 2005.

SALAY, E. Informação nutricional e mercado: um desafio para o governo e a universidade. Disponível em: <http://www.unicamp. br/imprensa>. Acesso em: 28 nov. 2003.

SALAY, E. Serviços de alimentação: crescimento acelerado. Agroanalysis, v. 21, n. 2, p. 47-49, 2001.

SLOAN, M. E.; BELL, L. N. Fat content of restaurant meals: comparison between menu and experimental values. Journal of the American Dietetic Association, v. 99, n. 6, p. 731-733, 1999.

SNEED, J.; BURKHALTER, J. P. Marketing nutrition in restaurants: a survey of current practices and attitudes. Journal of the American Dietetic Association, v. 91, n. 4, p. 459-462, 1991.

SBC/FUNCOR - SOCIEDADE BRASILEIRA DE CARDIOLOGIA/ FUNDO DE APERFEIÇOAMENTO E PESQUISA EM CARDIOLOGIA. Educação alimentar começa na infância. Disponível em: <http://www.cardiol.br>. Acesso em: 08 out. 2003.

STEWART, H.; BLISARD, N.; JOLLIFFE, D. Let's eat out: americans weigh taste, convenience, and nutrition. United States: United States Department of Agriculture - USDA. Disponível em: <http://www. ers.usda.gov>. Acesso em: 18 ago. 2006.

TARGET MARKETING. Brasil em Foco - IPC TARGET 2006 Disponível em: <http://www.targetmark.com.br>. Acesso em: 26 jul. 006. 
U.S. DEPARTMENT OF COMMERCE ECONOMICS AND STATISTICS ADMINISTRATION. U.S. CENSUS BUREAU. 1997 Economic Census. Accommodation and Foodservices. (Subject Series, Jul. 19, 2000).

VIGILÂNCIA SANITÁRIA DO MUNICÍPIO DO RIO DE JANEIRO. Informação nutricional nas redes de fast-food. Disponível em: $<$ http://www.rio.rj.gov.br/vigilanciasanitaria>. Acesso em: 20 out. 2005.
WEIMER, J. Accelerating the trend toward healthy eating. In: FRAZAO, E. (ed.) America's Eating Habits: changes and consequences. Washington, D.C.: United States Department of Agriculture USDA/Economic Research Service - ERS, 1999. Cap. 19, p. 385-401 (Agriculture Information Bulletin, n. 750). 